\title{
Prediction of robust stability boundaries for milling operations with extended multi-frequency solution and structured singular values
}

\author{
David Hajdu ${ }^{1 *}$, Tamas Insperger ${ }^{2 *}$, Daniel Bachrathy ${ }^{1 *}$, Gabor Stepan ${ }^{1 *}$ \\ ${ }^{1}$ Budapest University of Technology and Economics, Budapest, Hungary \\ ${ }^{2}$ Department of Applied Mechanics, Budapest University of Technology and Economics and MTA-BME Lendület Human Balancing Research Group, Budapest, \\ Hungary
}

\begin{abstract}
Reliable prediction of machine tool chatter is an essential problem in efficiency-oriented machine tool centers, since it requires the precise characterization of the dynamics of the machine-tool-workpiece system and the cutting force characteristics. Due to imperfect measurements, noise, uncertain and varying operational conditions, the mathematical models provide a deficient representation of the system. This leads to the need for the adaptation of robust stability analysis methods, which guarantee stability against bounded uncertainties and perturbations. In this paper, a frequency-domain approach is presented to calculate the robust stability boundaries of chatter-free machining parameters for milling operations. The idea is based on the concept of the stability radius and structured singular values, which is combined with the extended multi frequency solution. The proposed method is tested in a real case study.
\end{abstract}

Keywords: Milling, chatter, stability lobe diagram, robustness, structured singular values

\section{Introduction}

Industrial manufacturing experiences an increasing competition due to the recent development of powerful machining centers equipped with high-speed spindles and robust slide ways. Optimization of machining processes is an indispensable objective of the efficiency oriented industry. One of the strongest limitation in the industrial utilization of these high-performance machines is the undesired and harmful self-excited vibration, called machine tool chatter, that spoils the surface quality, increases the toolwear and reduces the life-time of the machine components. Reliable prediction of these vibrations is therefore an important task for manufacturing engineers.

The first mathematical models dealing with the self-excited vibrations in machining operations appeared in the work of Tobias [1] and Tlusty [2] in the 1950s and 1960s. After their pioneering research, the so-called regenerative effect became the most commonly accepted explanation for machine tool chatter. During the manufacturing process the vibrating tool leaves a wavy surface behind, which affects the chip thickness and induces variation in the cutting-force one revolution later. From the dynamic system's point of view, chatter is associated with the loss of stability of the stationary (chatter-free) machining process followed by a large amplitude self-excited vibration between the tool and the workpiece.

\footnotetext{
${ }^{*}$ Corresponding author

Email address: hajdu@mm. bme.hu (David Hajdu ${ }^{1}$ )
}

The stability properties of machining processes are depicted by the so-called stability lobe diagrams, which plot stable (chatter-free) domains in the plane of technological parameters (usually the spindle speed and the depth of cut). These diagrams provide a guide to the machinists to select optimal machining parameters and to avoid undesired vibrations.

There exist several mathematical methods to analyze the stability properties of machining operations and to construct stability lobe diagrams. Some of them apply the measured frequency response functions (FRFs) directly, such as the singlefrequency solution or zero-order approximation (ZOA), the multi-frequency solution (MFS) [3, 4] or the extended multifrequency solution (EMFS) [5]. Other time-domain based techniques, such as the semi-discretization method [6, 7], the fulldiscretization method [8], the integration method [9] and their extension by the implicit subspace iteration method [10], the Chebyshev collocation method [11, 12] and the spectral element method [13], require fitted modal parameters as input. In spite of the large number of available numerical methods, application of stability lobe diagrams is still not considered to be an essential element of machining. The primary reason for this is that the prediction of chatter-free technological parameters is not reliable enough to convince decision-makers. The input data used for the stability analysis, namely, the dynamics of the machine tool center and the parameters of the chip removal process model, contain many uncertainties and are loaded with measurement noise. These uncertainties together with model reductions and simplifications lead to an impaired representation of the real dynamical system.

Due to its simplicity, impact test is one of the most commonly 
used method to characterize the dynamics of the machine tool system and to obtain the frequency response functions. Dynamic measurements by impact tests are, however, affected by several uncertain factors: statistical variations, imperfect calibration coefficients for the hammer and transducer or misalignment between the intended and actual force direction during impact. For a detailed uncertainty analysis of measured FRFs see [14, 15].

Despite the need for a reliable method to predict robust stability lobe diagrams, only a few work have been published in this topic. The edge theorem combined with the zero exclusion method is presented in [16, 17] and compared to the results obtained by linear matrix inequalities in [18]. A robust chatter prediction method (RCPM) is introduced in [19], which applies a probabilistic approach and considers the parameters as random variables. A different concept based on fuzzy stability analysis is detailed in [20]. The above mentioned techniques require fitted modal parameters (and cutting parameters) as input variables, and the calculation time significantly increases with the number of uncertainties, therefore most of them are limited to systems with few uncertain parameters. In [21], an approximating numerical method is proposed, which provides confidence levels of stability boundaries for higher number of uncertain parameters. Robust stability analysis of turning processes is presented in [22] by means of envelope fitting around the measured FRFs combined with the single-frequency method. This method, however, cannot be applied to time-periodic processes, such as milling operations.

This paper presents a completely frequency-based solution for the robust stability analysis of milling processes, which utilizes directly the uncertainty of the measured frequency response functions and requires no fitted modal parameters. The stability analysis for milling operations is based on the extended multi-frequency solution [4, 5], while the robust stability analysis is applied according to the concept of structured singular values ( $\mu$-values) [23, 24]. The presented algorithm is able to generate robust stability lobe diagrams in reasonable time, which is an advantage in industrial applications.

The structure of the paper is as follows. In Section 2 the dynamical model of milling is introduced. Section 3 gives a detailed description on the stability analysis in frequency domain in a form, which is suitable for the robust stability analysis of the system. The structured singular value calculation is presented in Section 4 . The combination of these two concepts gives the new results in Section 5, which also highlights several numerical issues to solve the problem efficiently. The method is tested in a real case study in Section 6. The results are concluded in Section 7 .

\section{Dynamical model of milling}

In this section, cutting force model is presented for conventional helical milling tools with uniform helix angle, which are the most often used type of tools in the industry. Note, however, that the methods introduced in this paper can be extended to tools with nonuniform helix angles [25], variable pitch [26, 27], serrated cutter and distributed delay models [28], too.
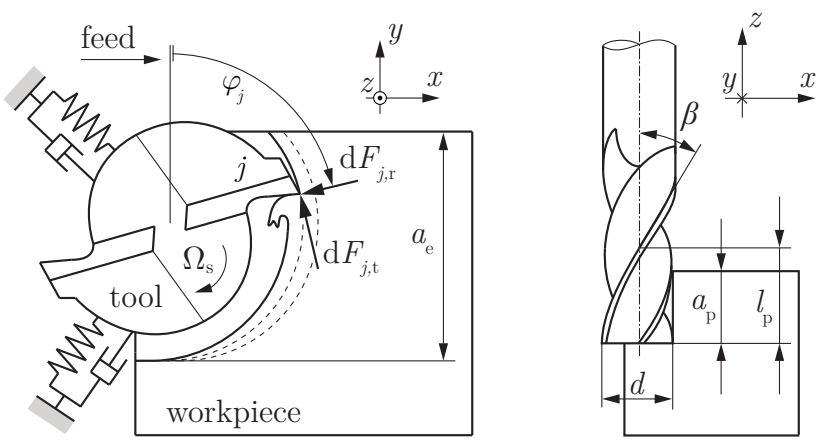

Figure 1: Dynamical model of milling with rigid workpiece and compliant tool.

The helical tool shown in Figure 1 has $N$ teeth of uniform helix angle $\beta$. According to [7], the tool is divided into elementary disks along the axial direction. The relation between the helix angle $\beta$, diameter $d$ and the helix pitch $l_{\mathrm{p}}$ is $\tan \beta=d \pi /\left(N l_{\mathrm{p}}\right)$, thus the angular position of the cutting edges along the axial direction reads

$$
\varphi_{j}(t, z)=\frac{2 \pi \Omega_{\mathrm{s}}}{60} t+j \frac{2 \pi}{N}-z \frac{2 \pi}{N l_{\mathrm{p}}},
$$

where $z$ is the coordinate along the axial immersion and $\Omega_{\mathrm{s}}$ is the spindle speed given in rpm. The elementary cutting-force components in tangential and radial directions acting on tooth $j$ at a disk element of width $\mathrm{d} z$ are

$$
\begin{aligned}
& \left.\mathrm{d} F_{j, \mathrm{t}}(t, z)=g_{j}(t, z)\left(K_{\mathrm{t}, \mathrm{e}}+K_{\mathrm{t}, \mathrm{c}} h_{j}(t, z)\right)\right) \mathrm{d} z, \\
& \left.\mathrm{~d} F_{j, \mathrm{r}}(t, z)=g_{j}(t, z)\left(K_{\mathrm{r}, \mathrm{e}}+K_{\mathrm{r}, \mathrm{c}} h_{j}(t, z)\right)\right) \mathrm{d} z,
\end{aligned}
$$

where $h_{j}(t, z)$ is the chip thickness cut by tooth $j$ at axial immersion $z, K_{\mathrm{t}, \mathrm{e}}$ and $K_{\mathrm{r}, \mathrm{e}}$ are the tangential and radial edge force coefficients, $K_{\mathrm{t}, \mathrm{c}}$ and $K_{\mathrm{r}, \mathrm{c}}$ are cutting force coefficients [29]. The screen function $g_{j}(t, z)$, which indicates whether the cutting edge is in contact with the material or not, reads

$$
g_{j}(t, z)= \begin{cases}1, & \text { if } \varphi_{\mathrm{en}}<\left(\varphi_{j}(t, z) \bmod 2 \pi\right)<\varphi_{\mathrm{ex}} \\ 0, & \text { otherwise }\end{cases}
$$

where $\varphi_{\mathrm{en}}$ and $\varphi_{\mathrm{ex}}$ are the entry and the exit immersion angles.

The position vector of the center of the tool-tip at time $t$ is denoted by $\mathbf{r}(t)=(x(t) y(t))^{\top}$. The actual chip thickness at tooth $j$ then can be calculated approximately as

$$
h_{j}(t, z) \approx\left(\mathbf{f}_{\mathrm{z}}+\mathbf{r}(t)-\mathbf{r}(t-\tau)\right)^{\top}\left(\begin{array}{c}
\sin \varphi_{j}(t, z) \\
\cos \varphi_{j}(t, z)
\end{array}\right),
$$

where vector $\mathbf{f}_{\mathrm{z}}=\left(f_{\mathrm{z}} 0\right)^{\top}$ describes the feed per tooth in direction $x$, and the tooth-passing period in case of constant pitch angle is $\tau=60 /\left(N \Omega_{\mathrm{s}}\right)$. The resultant cutting force vector $\mathbf{F}(t)=\left(F_{x}(t) F_{y}(t)\right)^{\top}$ can be calculated as

$$
\mathbf{F}(t)=-\sum_{j=1}^{N} \int_{0}^{a_{\mathrm{p}}} \mathbf{T}_{j}(t, z)\left(\begin{array}{l}
K_{\mathrm{t}, \mathrm{e}}+K_{\mathrm{t}, \mathrm{c}} h_{j}(t, z) \\
K_{\mathrm{r}, \mathrm{e}}+K_{\mathrm{r}, \mathrm{c}} h_{j}(t, z)
\end{array}\right) g_{j}(t, z) \mathrm{d} z,
$$


where the transformation matrix is

$$
\mathbf{T}_{j}(t, z)=\left(\begin{array}{cc}
\cos \varphi_{j}(t, z) & \sin \varphi_{j}(t, z) \\
-\sin \varphi_{j}(t, z) & \cos \varphi_{j}(t, z)
\end{array}\right)
$$

Assuming small perturbation $\boldsymbol{\varepsilon}(t)$ about the periodic motion $\mathbf{r}_{\mathrm{p}}(t)=\mathbf{r}_{\mathrm{p}}(t+\tau)$ of the stationary cutting, i.e. $\mathbf{r}(t)=\mathbf{r}_{\mathrm{p}}(t)+\boldsymbol{\varepsilon}(t)$, the cutting force can be expanded as

$$
\mathbf{F}(t)=\left.\mathbf{F}(t)\right|_{\mathbf{r}_{\mathrm{p}}(t)}+\left.\mathbf{D}_{\mathbf{r}(t)} \mathbf{F}(t)\right|_{\mathbf{r}_{\mathrm{p}}(t)}(\boldsymbol{\varepsilon}(t)-\boldsymbol{\varepsilon}(t-\tau)),
$$

where $\mathbf{D}_{\mathbf{r}(t)}$ is the gradient w.r.t. $\mathbf{r}(t)$ [25]. Derivation for nonlinear cutting force characteristics and generalized milling tools are presented in [25, 28]. In order to simplify the notation, the directional matrix is introduced as

$$
\mathbf{G}(t):=\left.\mathbf{D}_{\mathbf{r}(t)} \mathbf{F}(t)\right|_{\mathbf{r}_{\mathbf{p}}(t)},
$$

which is time-periodic with period $\tau$ [7].

\section{Extended multi-frequency solution}

Stability analysis of milling operations in frequency domain is an elegant approach, since no modal parameter fitting is required and direct application of measured frequency response functions is possible. One of the first frequency-domain method was the zero-order approximation (ZOA) proposed in [3], which approximates the periodic coefficients with constant averaged terms. This approximation, however, leads to imperfect stability charts for highly interrupted cutting such as low immersion milling. The ZOA method was improved to the multi-frequency solution (MFS) in [4], where the higher Fourier harmonics in the periodic terms were also considered. In order to reduce the computational effort, the extended multifrequency solution (EMFS) was presented in [5]. In this section, the EMFS technique is presented briefly for single point delay, which is then extended to robust stability analysis in the subsequent sections.

The dynamic behavior of the system in the frequency domain is represented by

$$
\mathbf{r}(\omega)=\mathbf{H}(\omega) \mathbf{F}(\omega),
$$

where $\mathbf{r}(\omega)$ is the Fourier transform $\mathcal{F}$ of the position vector $\mathbf{r}(t), \mathbf{F}(\omega)$ is the Fourier transform of the force vector $\mathbf{F}(t)$, while $\mathbf{H}(\omega)$ is the frequency response function matrix of the tool [30]. During the stability analysis, the periodic motion can be separated, and only the stability of the perturbed motion $\boldsymbol{\varepsilon}(t)=\mathbf{r}(t)-\mathbf{r}_{\mathrm{p}}(t)$ about the periodic orbit has to be considered. The Fourier transform of 8 with the use of 10 and after simplification with the stationary part yield

$$
\boldsymbol{\varepsilon}(\omega)=\mathbf{H}(\omega) \mathbf{G}(\omega) *\left(\boldsymbol{\varepsilon}(\omega)\left(1-\mathrm{e}^{-\mathrm{i} \omega \tau}\right)\right),
$$

where $*$ denotes the convolution and $\mathbf{G}(\omega)$ is the Fourier transform of $\mathbf{G}(t)$. According to the Floquet theory [31], the solution at the stability limit has the trial form

$$
\boldsymbol{\varepsilon}(t)=\mathbf{p}(t) \mathrm{e}^{\mathrm{i} \omega_{\mathrm{c}} t}, \mathbf{p}(t)=\mathbf{p}(t+\tau),
$$

where $\omega_{\mathrm{c}}$ is the chatter frequency. The periodic functions can be expanded to Fourier series and can be represented by Dirac delta functions $\delta(\omega)$ in the frequency domain as

$$
\begin{aligned}
\mathbf{p}(\omega) & =\sum_{k=-\infty}^{\infty} \mathbf{p}_{k} \delta(\omega-k \Omega), \\
\mathcal{F}\left(\mathrm{e}^{\mathrm{i} \omega_{\mathrm{c}} t}\right) & =\delta\left(\omega-\omega_{\mathrm{c}}\right) \\
\mathbf{G}(\omega) & =\sum_{l=-\infty}^{\infty} \mathbf{G}_{l} \delta(\omega-l \Omega),
\end{aligned}
$$

where indices $k$ and $l$ refer to the corresponding Fourier components and $\Omega=2 \pi / \tau$. Then (11) can be reformulated as

$$
\begin{array}{r}
\sum_{k=-\infty}^{\infty} \mathbf{p}_{k} \delta\left(\omega-k \Omega-\omega_{\mathrm{c}}\right)=\mathbf{H}(\omega) \sum_{l=-\infty}^{\infty} \mathbf{G}_{l} \delta(\omega-l \Omega) \\
* \sum_{k=-\infty}^{\infty} \mathbf{p}_{k} \delta\left(\omega-k \Omega-\omega_{\mathrm{c}}\right)\left(1-\mathrm{e}^{-\mathrm{i} \omega \tau}\right) .
\end{array}
$$

Using the shifting theorem in the form

$$
\delta(\omega-l \Omega) * \delta\left(\omega-k \Omega-\omega_{\mathrm{c}}\right)=\delta\left(\omega-(l+k) \Omega-\omega_{\mathrm{c}}\right),
$$

the convolution of two discrete series can be rearranged as a Cauchy product and $(16)$, after simplification with the indices, can be written as

$$
\begin{aligned}
\sum_{k=-\infty}^{\infty} \mathbf{p}_{k} \delta(\omega- & \left.k \Omega-\omega_{\mathrm{c}}\right)=\mathbf{H}(\omega) \sum_{l=-\infty}^{\infty} \sum_{k=-\infty}^{\infty} \mathbf{G}_{l-k} \\
& \times \mathbf{p}_{k} \delta\left(\omega-l \Omega-\omega_{\mathrm{c}}\right)\left(1-\mathrm{e}^{-\mathrm{i} \omega \tau}\right) .
\end{aligned}
$$

The sampling effect of the Dirac delta functions on $\mathbf{H}(\omega)$ and $\mathrm{e}^{-\mathrm{i} \omega \tau}$ leads to

$$
\begin{aligned}
& \sum_{k=-\infty}^{\infty} \mathbf{p}_{k} \delta(\omega-\left.k \Omega-\omega_{\mathrm{c}}\right)=\sum_{l=-\infty}^{\infty} \sum_{k=-\infty}^{\infty} \mathbf{H}\left(l \Omega+\omega_{\mathrm{c}}\right) \mathbf{G}_{l-k} \\
& \times \mathbf{p}_{k} \delta\left(\omega-l \Omega-\omega_{\mathrm{c}}\right)\left(1-\mathrm{e}^{-\mathrm{i}\left(l \Omega+\omega_{\mathrm{c}}\right) \tau}\right) .
\end{aligned}
$$

The equality holds for all Dirac delta functions. This results an infinite-dimensional system in the form

$$
\left(\begin{array}{c}
\vdots \\
\mathbf{p}_{-1} \\
\mathbf{p}_{0} \\
\mathbf{p}_{1} \\
\vdots
\end{array}\right)=\underbrace{\left(\begin{array}{lllll}
\ddots & & & & \\
& \mathbf{A}_{-1,0} & \mathbf{A}_{-1,-1} & \mathbf{A}_{-1,-2} & \\
& \mathbf{A}_{0,1} & \mathbf{A}_{0,0} & \mathbf{A}_{0,-1} & \\
& \mathbf{A}_{1,2} & \mathbf{A}_{1,1} & \mathbf{A}_{1,0} & \\
& & & & \ddots
\end{array}\right)}_{=: \mathbf{Q}_{\infty}} \underbrace{\left(\begin{array}{c}
\vdots \\
\mathbf{p}_{-1} \\
\mathbf{p}_{0} \\
\mathbf{p}_{1} \\
\vdots
\end{array}\right)}_{=: \mathbf{u}_{\infty}},
$$

where

$$
\mathbf{A}_{m, n}=\mathbf{H}\left(m \Omega+\omega_{\mathrm{c}}\right) \mathbf{G}_{n}\left(1-\mathrm{e}^{-\mathrm{i}\left(m \Omega+\omega_{\mathrm{c}}\right) \tau}\right) .
$$

The infinite dimensional matrix $\mathbf{Q}_{\infty}$ is a variation of Hill's infinite matrix [32], which can be approximated by truncation to the first $\pm r$ frequency components to get the truncated matrix 
$\mathbf{Q} \in \mathbb{C}^{2(2 r+1) \times 2(2 r+1)}$ and vector $\mathbf{u} \in \mathbb{C}^{2(2 r+1)}$. The existence of a periodic solution about the stationary motion at some $\omega_{\mathrm{c}}$ requires that

$$
(\mathbf{I}-\mathbf{Q}) \mathbf{u}=\mathbf{0},
$$

that is,

$$
\operatorname{det}(\mathbf{I}-\mathbf{Q})=0,
$$

where $\mathbf{I}$ is the identity matrix with the appropriate dimensions. Note, that $\mathbf{Q}$ is a function of $\omega_{\mathrm{c}}$, which is omitted in order to simplify the notation. The chatter frequency therefore has to be analyzed over a given interval to check whether 23 is satisfied or not. In this form, the solution can be applied for any tool geometry and any tool dynamics described by the formulas above, see [5, 25].

Note, that the truncated Hill's matrix can be represented as a product of matrices as

$$
\mathbf{Q}=\mathbf{U E W},
$$

where the decomposed matrices separate the dynamics, the regenerative delay and the force model. The structures are

$$
\begin{aligned}
& \mathbf{U}=\oplus_{m=-r}^{r} \mathbf{H}\left(m \Omega+\omega_{\mathrm{c}}\right), \\
& \mathbf{E}=\oplus_{m=-r}^{r} \mathbf{I}\left(1-\mathrm{e}^{-\mathrm{i}\left(m \Omega+\omega_{\mathrm{c}}\right) \tau}\right), \\
& \mathbf{W}_{m, n}=\mathbf{G}_{m-n}, \quad m, n \in[-r, r]
\end{aligned}
$$

where $\mathbf{W}$ is a Toeplitz matrix with elements $\mathbf{G}_{j}$, and $\oplus$ denotes the matrix direct sum, i.e.

$$
\oplus_{j=-r}^{r} \mathbf{A}_{j}=\operatorname{diag}\left(\mathbf{A}_{-r}, \ldots, \mathbf{A}_{r}\right) .
$$

Similarly to $\mathbf{Q}$, matrices $\mathbf{U}$ and $\mathbf{E}$ also depend on the chatter frequency $\omega_{\mathrm{c}}$, although the notation is omitted for the sake of simplicity. The stability boundaries for the system with deterministic parameters can be determined using equation (23). In the next sections, the robust stability analysis in case of uncertain frequency response functions is described.

\section{Robust analysis}

Analysis of robustness of systems with respect to parametric uncertainties is typically a time-consuming process and evaluation time significantly depends on the number of uncertain variables. Methods such as the edge theorem combined with zero exclusion [16, 17] or the RCPM [19] are efficient tools for low number of parameters only. Let us use the notation $\delta$ for the perturbations. For instance, perturbation of the FRF matrix $\mathbf{H}(\omega)$ is $\delta \mathbf{H}(\omega)$ and the perturbed FRF matrix is $\mathbf{H}(\omega)+\delta \mathbf{H}(\omega)$. Then the truncated Hill's matrix can be written as

$$
\mathbf{Q}+\delta \mathbf{Q}=(\mathbf{U}+\delta \mathbf{U}) \mathbf{E W}
$$

where

$$
\begin{aligned}
\delta \mathbf{Q} & =\delta \mathbf{U E W}, \\
\delta \mathbf{U} & =\oplus_{m=-r}^{r} \delta \mathbf{H}\left(m \Omega+\omega_{\mathrm{c}}\right) .
\end{aligned}
$$

Note, that the structure of $\delta \mathbf{U}$ is constrained to block-diagonal matrices. Inserting the perturbation (27) into 23 yields

$$
\operatorname{det}(\mathbf{I}-(\mathbf{U}+\delta \mathbf{U}) \mathbf{E W})=0 .
$$

Robustness of the system can be measured by finding the smallest $\delta \mathbf{U}$ from the possible perturbations such that (30) holds. For this analysis, the concept of structured singular values is used, which is introduced briefly in the next subsection.

\subsection{Introduction of structured singular values}

The original concept of structured singular values ( $\mu$-values) was introduced in [23] to analyze the effect of block-diagonal perturbations of matrices. Since its first application, the method was found to be an effective tool in the analysis of robustness of dynamic systems subjected to perturbations or in the control design for systems with structured and unstructured uncertainties [24].

Let us consider a general matrix $\mathbf{M} \in \mathbb{C}^{m \times n}$, a perturbation set $\boldsymbol{\Delta}_{\mathrm{s}} \in \mathbb{C}^{n \times m}$ and a given norm $\|\cdot\|$. Then the $\mu$-value of $\mathbf{M}$ is the inverse of the smallest $\|\boldsymbol{\Delta}\|, \boldsymbol{\Delta} \in \boldsymbol{\Delta}_{\mathrm{s}}$, such that 1 is an element of the spectrum of the matrix product $\Delta \mathbf{M}$ (see [24, 33]). An equivalent definition, which is more meaningful in our context, says that the $\mu$-value of matrix $\mathbf{M}$ is the inverse of the smallest perturbation such that $\operatorname{det}(\mathbf{I}-\mathbf{\Delta} \mathbf{M})=0$, i.e.

$$
\mu(\mathbf{M})=\left(\inf \left\{\|\mathbf{\Delta}\|, \Delta \in \Delta_{\mathrm{s}}, \operatorname{det}(\mathbf{I}-\mathbf{\Delta} \mathbf{M})=0\right\}\right)^{-1} .
$$

Note, that the larger the $\mu$-value is, the less robust the system is.

The definition of $\mu$, however, does not provide any calculation method. Formulas for the determination of $\mu$ in case of complex perturbations are presented in [33, 34], and for real cases in [33, 35], just to mention a few.

Precise calculation of structured singular values, however, is based on the solution of a convex optimization problem solved in the form of linear matrix inequalities [34], therefore it can be extremely time-consuming for large dimensions.

Unfortunately, it is also shown that the exact $\mu$-values cannot be determined exactly for arbitrary perturbation structures [23]. Instead, an upper and a lower bounds can calculated, which might also be suitable for practical applications.

In order to provide an efficient formula for the characterization of the robustness of milling operations, a fast and efficient technique is required. In the forthcoming part the concept introduced in [24] is presented briefly, then its application for milling operations is detailed in Section 5

Let us introduce a real-valued weight matrix $\mathbf{R}=\left[R_{j, k}\right]$, $R_{j, k} \geq 0, \forall j, k$ (a non-negative matrix) and a weighted maximum norm defined as

$$
\|\Delta\|_{\mathrm{m}}:=\max _{j, k} R_{j, k}^{-1}\left|\Delta_{j, k}\right|
$$

where subscript $\mathrm{m}$ refers to the maximum norm. In this case the following equivalence holds for $\boldsymbol{\Delta}$

$$
\|\boldsymbol{\Delta}\|_{\mathrm{m}} \leq 1 \quad \Leftrightarrow \quad\left|\Delta_{j, k}\right| \leq R_{j, k}, \forall j, k .
$$


Consequently, if $\|\boldsymbol{\Delta}\|_{\mathrm{m}}=1$, then there exists at least (but not necessarily) one element in matrix $\mathbf{R}$ such that $\left|\Delta_{j, k}\right|=R_{j, k}$. Then an upper bound on $\mu$-values can be given by the relations

$$
\mu(\mathbf{M})=\max _{\|\boldsymbol{\Delta}\|_{\mathrm{m}}=1} \rho(\boldsymbol{\Delta} \mathbf{M}) \leq \rho(\mathbf{R} \tilde{\mathbf{M}})=\rho(\tilde{\mathbf{M}} \mathbf{R}),
$$

where $\rho$ denotes the spectral radius, and $\tilde{\mathbf{M}}$ is a non-negative matrix with $\tilde{M}_{j, k}=\left|M_{j, k}\right|$ (see [24]). It is also shown in [24], that the $\mu$-value and the so-called stability radius $r_{\mathbb{C}}$ of a characteristic matrix $\mathbf{P}(\omega)$ have the connection

$$
r_{\mathbb{C}}=\left(\sup _{\omega \geq 0} \mu(\mathbf{P}(\omega))\right)^{-1},
$$

where the stability radius gives a measure from the distance of instability. If $r_{\mathbb{C}}=0$, then the system is on the boundary of stability, while as $r_{\mathbb{C}} \rightarrow \infty$, the system guarantees stability against infinitely large uncertainties defined by the perturbation structure $\boldsymbol{\Delta}_{\mathrm{s}}$. It is important to note, that at $r_{\mathbb{C}}=1$ there exists a perturbation matrix, which just drifts the system to the boundary of stability, in other words, the system is on the boundary of robust stability.

Detailed derivations, theorems and proofs related to the topic for general cases can be found in [24]. An important note is that the inequality in (34) is actually an equality if $\mathbf{M}$ is a diagonal matrix (see [24]).

\section{Application of structured singular values in milling}

Analysis of robustness of milling operations requires the representation of the governing equation in the $\mathbf{\Delta M}$ structure, and the determination of bounds for the uncertainty denoted by matrix $\mathbf{R}$.

If the perturbation of the frequency response functions is bounded along the frequency, then there exists $\delta \tilde{\mathbf{H}}(\omega)$ matrix, such that

$$
0 \leq\left|\delta H_{j, k}(\omega)\right| \leq \delta \tilde{H}_{j, k}(\omega), \quad j=1,2, k=1,2, \omega \geq 0,
$$

where indices represent the elements of the frequency response function matrix. Note, that $H_{j, k}(\omega)$ is a complex, while $\delta \tilde{H}_{j, k}(\omega)$ is a positive real function. Therefore, the perturbations $\delta H_{j, k}(\omega)$ can be represented by disks with varying radius $\delta \tilde{H}_{j, k}(\omega)$ around the graph of $H_{j, k}(\omega)$ along the frequency $\omega$.

The perturbed governing equation (see 30) implies

$$
(\mathbf{I}-(\mathbf{U}+\delta \mathbf{U}) \mathbf{E W}) \mathbf{u}=\mathbf{0},
$$

then some algebraic manipulation gives

$$
\mathbf{u}=(\mathbf{I}-\mathbf{U E W})^{-1} \delta \mathbf{U E W u} .
$$

Multiplication of both sides by $\mathbf{E W}$, introducing new variable $\mathbf{v}=\mathbf{E W u}$ and simplification by (24) gives

$$
\mathbf{v}=\mathbf{E W}(\mathbf{I}-\mathbf{Q})^{-1} \delta \mathbf{U v},
$$

from which a standard form is obtained after bringing all the terms to the left-hand side

$$
\left(\mathbf{I}-\mathbf{E W}(\mathbf{I}-\mathbf{Q})^{-1} \delta \mathbf{U}\right) \mathbf{v}=\mathbf{0} .
$$

The nontrivial solution of the equation implies that

$$
\operatorname{det}\left(\mathbf{I}-\mathbf{E W}(\mathbf{I}-\mathbf{Q})^{-1} \delta \mathbf{U}\right)=0 .
$$

Clearly, this is identical to the $\Delta \mathbf{M}$ structure, where $\boldsymbol{\Delta}=\delta \mathbf{U}$ and $\mathbf{M}=\mathbf{E W}(\mathbf{I}-\mathbf{Q})^{-1}$. Note that the opposite order of the multiplication of the terms $\mathbf{M}$ and $\boldsymbol{\Delta}$ does not affect the determinant and the spectrum due to Sylvester's determinant identity. According to [24], an upper estimation on $\mu$ can be given by [34, where

$$
\tilde{M}_{j, k}=\left|\mathbf{E W}(\mathbf{I}-\mathbf{Q})^{-1}{ }_{j, k}\right|,
$$

and $\left|\delta U_{j, k}\right| \leq R_{j, k} \forall j, k$. Since the perturbation of the FRF matrix is bounded, the weight matrix $\mathbf{R}$ can be calculated as

$$
\mathbf{R}=\oplus_{m=-r}^{r} \delta \tilde{\mathbf{H}}\left(m \Omega+\omega_{\mathrm{c}}\right)
$$

Note again, that similarly to matrices $\mathbf{Q}, \mathbf{U}$ and $\mathbf{E}$, the weight matrix $\mathbf{R}$ and $\tilde{\mathbf{M}}$ are also functions of $\omega_{\mathrm{c}}$, therefore $\mu$ has to be evaluated along the chatter frequency. Finally the relation between the stability radius and $\mu$-values gives

$$
r_{\mathbb{C}}>\tilde{r}:=\left(\sup _{\omega_{\mathrm{c}} \geq 0} \rho(\tilde{\mathbf{M} R})\right)^{-1},
$$

where $\tilde{r}$ is a lower estimation of the stability radius, therefore it gives conservative estimation. The robust stability boundary is given by the level curve $\tilde{r}=1$.

Note, that the conservatism of the stability radius can be reduced, if the $\mu$-values are calculated with conventional algorithms (see. [34]), however, it might lead to extremely long computational time. A numerical study is given in the Appendix, comparing the proposed algorithm to the conventional method. Another way to reduce the difference between the calculated stability radius and real robust bound is to separate the real and imaginary parts of the frequency response function, and define the real- $\mu$ problem, see [36]. Again, an important drawback is the significant calculation effort required to obtain the robust stability boundaries even for the simplest models. In order to keep the computational effort at low cost, these extensions are omitted in this study and only the bound defined by Eq. 34 is used.

\subsection{Efficient calculation}

The computation of the robust stability boundary is always time-consuming compared to the stability analysis of the deterministic system. Therefore optimization of the numerical techniques is of high importance. In this subsection a few numerical issues are discussed.

The EMFS is an efficient method for the stability analysis of the deterministic system [5]. The stability boundaries in the plane of spindle speed and depth of cut can be determined if the chatter frequency $\omega_{\mathrm{c}}$ is considered as an extra third parameter of the stability chart. The sweeping range of the chatter frequency can be restricted to $\omega_{\mathrm{c}} \in[0, \Omega / 2]$, since $\mathbf{Q}$ is $\Omega$ periodic, that is, $\mathbf{Q}\left(\omega_{\mathrm{c}}\right)=\mathbf{Q}\left(\omega_{\mathrm{c}}+\Omega\right)$, and has the symmetry $\mathbf{Q}\left(\omega_{\mathrm{c}}\right)=\overline{\mathbf{Q}}\left(-\omega_{\mathrm{c}}\right)$. An effective tool to find the roots of 23) is the so-called multi-dimensional bisection method (MDBM) 

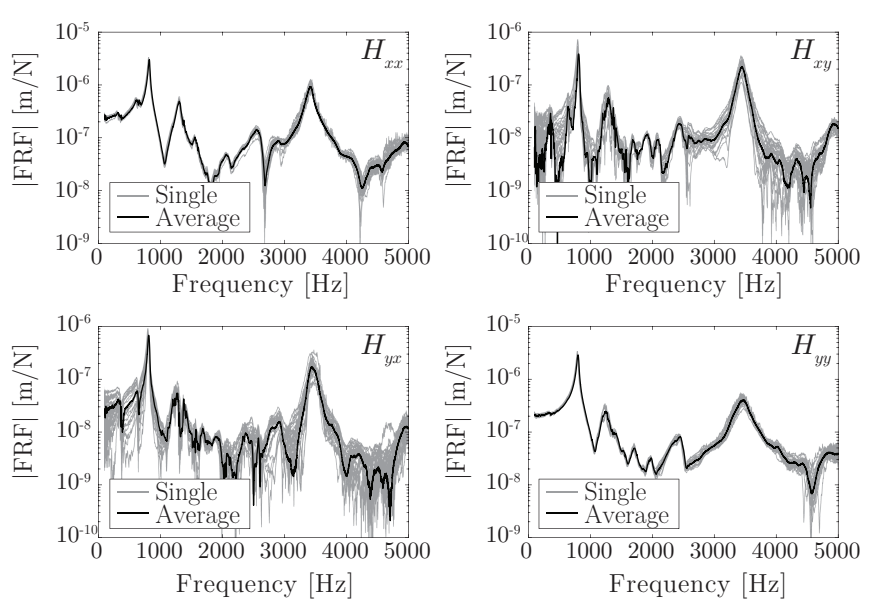

Figure 2: Measured frequency response functions.

[37]. Multiple boundary lines, closed curves of stable and unstable islands in the stability charts can all be found automatically using the MDBM. The efficiency of the method can be further increased if the required number of harmonics are limited according to the formula $r=\operatorname{ceil}\left(\omega_{\mathrm{n}}^{\max } \tau / \pi\right)$, where $\omega_{\mathrm{n}}^{\max }$ is the highest relevant natural angular frequency of the system [5].

The computation time of the robust stability boundary can be reduced using the above mentioned solutions; however, some important changes have to be made. One of the most important advantages of the EMFS is that it requires the computation of a determinant of a matrix, which is usually much faster than eigenvalue computations. According to (34), robust stability boundaries can be determined via computing the largest eigenvalue of the matrix product $\tilde{\mathbf{M R}}$. Since both matrices have nonnegative entries only, their product is a nonnegative matrix, too, therefore the Perron-Frobenius theorem can be applied. According to [38], a matrix A with entries $a_{j, k}>0$ always has a positive real eigenvalue $\gamma$ (called Perron root or the PerronFrobenius eigenvalue) which is larger in magnitude than any other eigenvalue. At the robust stability boundary it is known, that $\tilde{r}=\rho(\tilde{\mathbf{M} R})^{-1}=1$ at some $\omega_{\mathrm{c}}$, which condition can be reformulated as

$$
\operatorname{det}(\tilde{\mathbf{M}} \mathbf{R}-\mathbf{I})=0
$$

Note that this determinant is now a real number, therefore (45) gives a single scalar equation, as opposed to the MFS, where a complex valued determinant gives two independent scalar equations. The final condition, which has to be evaluated along the plane of the machining parameters $\left(\Omega_{\mathrm{s}}, a_{\mathrm{p}}\right)$ to find the robust boundary is

$$
\min _{\omega_{\mathrm{c}} \in[0, \Omega / 2]}(\operatorname{det}(\tilde{\mathbf{M}} \mathbf{R}-\mathbf{I}))=0
$$

where both $\tilde{\mathbf{M}}$ and $\mathbf{R}$ are non-negative real matrices and are functions of $\omega_{\mathrm{c}}$.

Since the extremum is sought along the chatter frequency $\omega_{\mathrm{c}}$, the function (45) should be numerically differentiated. This leads to noisy results when applied to measured FRFs directly. Therefore the chatter frequency should be swept numerically
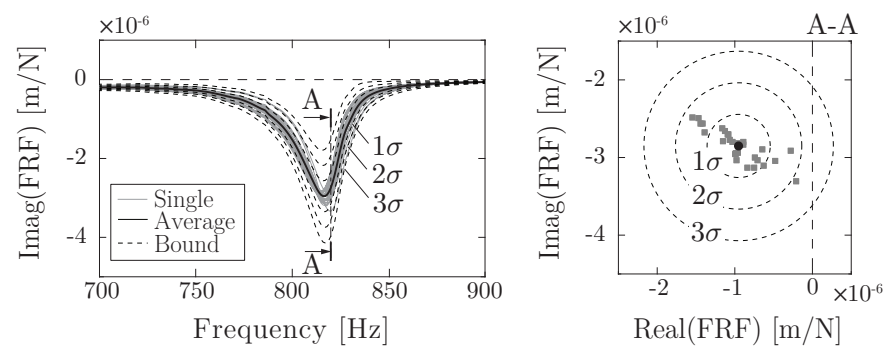

Figure 3: Envelope calculated from the variance of $H_{x x}(\omega)$.

with a sufficiently small frequency step $\mathrm{d} \omega$ from 0 to $\Omega / 2$ in order to find the minimum. The parameter space $\left(\Omega_{\mathrm{s}}, a_{\mathrm{p}}\right)$, however, can be iterated by the bisection method [37].

Another problem with the evaluation of the robust stability boundary is that, similarly to the EMFS, it does not distinguish the stable and unstable parameter domains. In order to avoid unnecessary computations in unstable regions, first the stability of the parameter point should be determined. Unstable parameter points can be excluded from the iteration using the Cauchy Argument Principle, similarly to the Nyquist criterion [5, 39]. Since the frequency is already swept with a fixed resolution, the number of unstable multipliers can be calculated. The determinant in 23 forms a closed parametric curve in the complex plane as a function of $\omega_{\mathrm{c}} \in[-\Omega / 2, \Omega / 2]$. If this curve has a positive number of clockwise encirclements of the origin then the system is unstable [5]. The number $P$ of poles along the interval $\omega_{\mathrm{c}} \in[-\Omega / 2, \Omega / 2]$ can be calculated as

$$
P=\frac{1}{\pi \mathrm{i}} \int_{0}^{\Omega / 2} \frac{D^{\prime}\left(\omega_{\mathrm{c}}\right)}{D\left(\omega_{\mathrm{c}}\right)} \mathrm{d} \omega_{\mathrm{c}},
$$

where

$$
D\left(\omega_{\mathrm{c}}\right)=\operatorname{det}\left(\mathbf{I}-\mathbf{Q}\left(\omega_{\mathrm{c}}\right)\right),
$$

$(\cdot)^{\prime}$ denotes differentiation with respect to $\omega_{\mathrm{c}}$ and $\mathrm{i}^{2}=-1$.

A flowchart is presented in the appendix in Figure 5, which gives the necessary steps to calculate the robustness of a single parameter point $\left(\Omega_{\mathrm{s}}, a_{\mathrm{p}}\right)$. In order to calculate the whole stability lobe diagram, the same process has to be evaluated along the points of the diagram.

\section{Experiment}

An experiment is carried out in an NCT EmR-610Ms type milling machine with a Tivoly $\mathrm{P} 615 \mathrm{H}$ tool. Modal experiments are performed at the tool-tip using a National Instruments data

Table 1: Parameters for the milling tests.

\begin{tabular}{ll}
\hline Tool & Tivoly P615H \\
& $N=2, d=16 \mathrm{~mm}, \beta=35^{\circ}$ \\
Workpiece & Aluminum 2024-T351 \\
& $K_{\mathrm{t}, \mathrm{e}}=13.9 \mathrm{~N} / \mathrm{mm}, K_{\mathrm{t}, \mathrm{c}}=1336 \mathrm{~N} / \mathrm{mm}^{2}$ \\
& $K_{\mathrm{r}, \mathrm{e}}=22.3 \mathrm{~N} / \mathrm{mm}, K_{\mathrm{r}, \mathrm{c}}=593 \mathrm{~N} / \mathrm{mm}^{2}$ \\
Process & Up-milling, $a_{\mathrm{e}}=1 \mathrm{~mm}, f_{\mathrm{z}}=0.05 \mathrm{~mm} /$ tooth \\
& $\left(\varphi_{\mathrm{en}}=\operatorname{acos}\left(2 a_{\mathrm{e}} / d-1\right), \varphi_{\mathrm{ex}}=\pi\right)$ \\
\hline
\end{tabular}


acquisition device, a Brüel\&Kjær 2302-10 impact hammer and a PCB acceleration sensor. The cutting parameters are fitted onto cutting experiments measured by a Kistler dynamometer. The measured material was an aluminum alloy 2024-T351. The theoretical parameters are listed in Table 1 .

During the modal analysis, the tool was measured five times both in the main- and in the cross directions. Then it was detached from the spindle, and then remounted to simulate the tool change procedure. The whole process was repeated six times. Note, that in order to emphasize the measurement uncertainty, each individual frequency response function was calculated from a single impact test without any additional averaging. The resulting transfer functions (30 in each case) and the average of those can be seen in Fig. 2. The actual dynamical properties of the complete machining system may vary with the spindle speed, ambient temperature, spindle temperature, etc. It is also observed that removing and replacing the tool modify slightly the tool-tip-dynamics as the micro contact area between the tool holder and the spindle changes. The dynamic stiffness may also vary slowly in time due to the uncertain contact area. All of these effects are not taken into account in the conventional mechanical models and averaging does not solve this issue since FRFs are measured typically before the cutting tests. Instead of considering the complex contact phenomena, we assume that the FRF is loaded by some static uncertainty. This is also relevant in the industry, since the tool cannot be continuously measured during machining operations.

If the uncertainties of the measured FRFs are known, then the weight matrix $\mathbf{R}$ can be constructed. Here we describe the uncertainties by the variance only (similarly to a normal distribution). The variance of the complex function is then calculated as

$$
\sigma(u(\omega)+\mathrm{i} v(\omega))=\sqrt{\sigma(u(\omega))^{2}+\sigma(v(\omega))^{2}} .
$$

An example is presented in Fig. 3, which shows the boundaries corresponding to $1 \sigma, 2 \sigma$ and $3 \sigma$. This figure also highlights the drawback of the method. During the calculation of the $\mu$-values, only a circular uncertainty can be considered, while the covariance between the real and imaginary values is omitted (for a related study, see [14]). Therefore the fitted envelope can be considered as a conservative estimation, which may unnecessarily overestimate uncertainty. If the distribution of the frequency response functions is normal, moreover the real and imaginary values are independent, then the boundary $1 \sigma$ includes approximately $68 \%$ of the points. This is, however, not true, in our case: the real and imaginary values are not independent but are distributed mainly along a line in the complex plane as it can be seen in Fig. 3. Therefore the application of $1 \sigma$ already gives a more conservative estimation than it would give for an ideal normal distribution with independent real and imaginary parts.

In order to compare the different scenarios, the robust stability lobe diagrams were calculated for uncertainty level $1 \sigma$, $2 \sigma$ and $3 \sigma$, respectively. The results of the computation can be seen in Fig. 4 a), where the nominal boundary is indicated by solid black line, the corresponding domain of stable machining parameters is denoted by gray shaded area, while the different robust stability boundaries corresponding to different levels
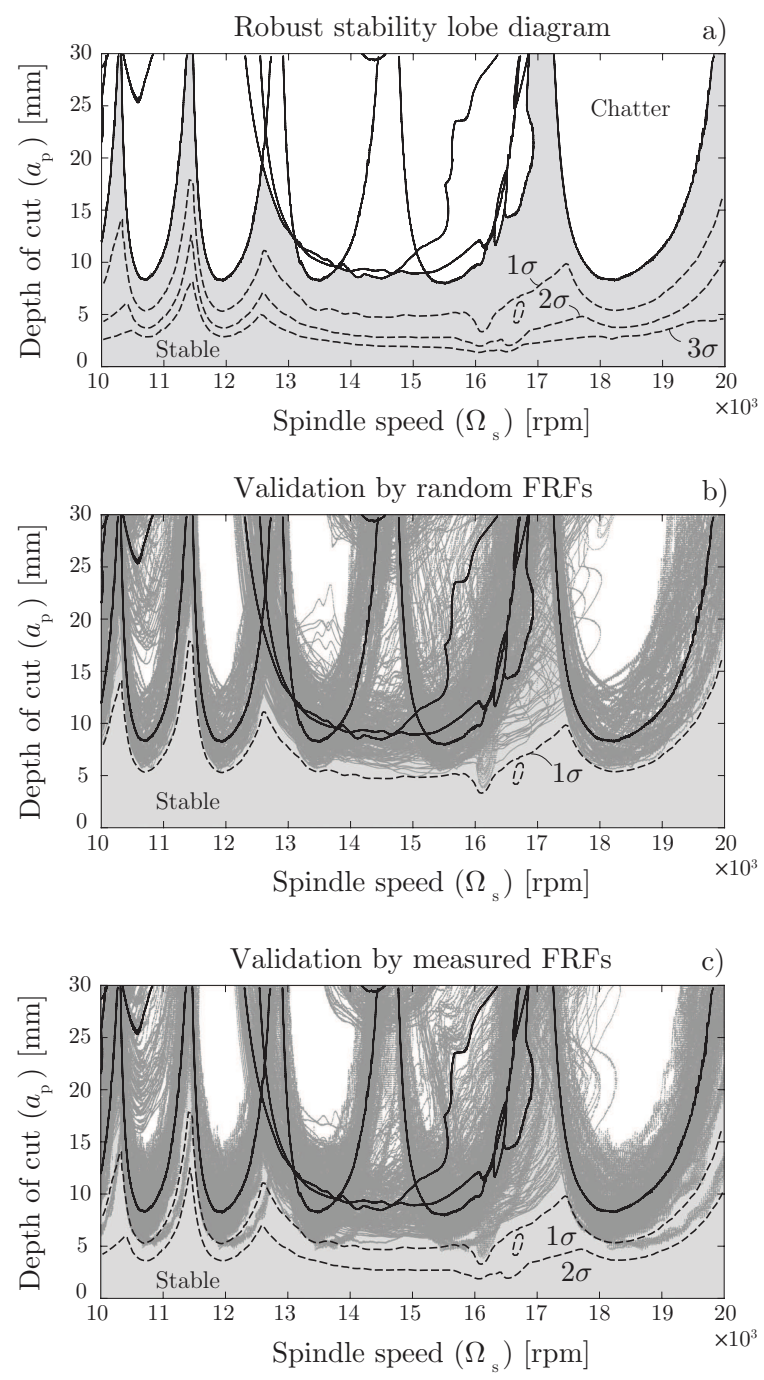

Figure 4: Case study: a) Conventional and robust stability boundary b) Validation of robust stability boundary c) Comparison with measured frequency response functions.

of uncertainties are indicated by dashed black lines. It can be seen that the sensitivity along the spindle speed is not uniform, some boundaries can change drastically if the FRF matrix is perturbed.

The calculations were carried out in MATLAB environment, simulation were run on a personal computer with Intel Core i74510U $2.00 \mathrm{GHz}$ CPU and 8 GB RAM.

In order to validate the accuracy of the numerical method, 100 random FRF matrices were generated numerically from the uncertain domain bounded by $1 \sigma$, and the corresponding stability lobe diagrams were calculated. The results are presented in Fig. 4 b), where the solid gray lines indicate the boundaries corresponding to the perturbed FRF matrices. The predicted robust stability lobe diagram bounds the perturbed curves, while the mismatch between them is not significant.

In Fig. 4 c) the effect of the real measured FRF matrices are compared to the predicted robust stability lobe diagrams. Since the frequency response functions were measured 30 times in each direction, the possible combination of the FRF matrix is 
$30^{4}=810000$. Clearly, all of those cannot be evaluated within reasonable time, therefore only 100 random combinations were selected.

The calculation of the corresponding 100 stability lobe diagrams took $28912 \mathrm{sec}$ (approx. 8 hours in total, $289 \mathrm{sec}$ for each), while the robust stability boundary was determined within 295 seconds (4.9 min).

The perturbed stability lobe diagrams, similarly to the previous case, are indicated by solid gray lines. It was found that the $1 \sigma$ level curve characterizes well the robustness of the measured system. There are only two FRF combinations, which results stability boundaries below the predicted robust boundary, these cases however have very small probability. Therefore in $98 \%$ of the calculations the predicted robust stability boundary with $1 \sigma$ uncertainty was found to be sufficient. The reason for this high percentage is that the circular bounds generally overestimate the actual uncertainty, while $1 \sigma$ underestimates it, as shown by Fig. 3. In case of more rigorous conditions, the level curve $2 \sigma$ might be considered.

\section{Conclusion}

The extended multi-frequency solution and the method of $\mu$ analysis were combined to determine robust stability boundaries for milling operations with uncertain dynamics. The measured FRFs can directly be applied, and no modal parameter fitting is required, which is an essential aspect for industrial applications [5].

Several numerical issues were discussed in order to speed up the computation significantly. Based on a series of numerical tests, it can be stated that under optimized resolutions and settings, the computational time of the robust stability boundaries is approximately 2-5 times longer than the calculation of the conventional stability lobe diagram.

The definition of stability radius in terms of the $\mu$-values was found to be an effective method in the analysis of sensitivity of milling operations with respect to FRF-based uncertainties. When the measured frequency response functions are loaded with significant noise, then uncertainty can be characterized by the variance of the measurements. The proposed concept utilizes directly the uncertainty of the transfer function matrix, which can be calculated during the sequentially repeated modal experiments.

The results were tested in a real case study and recommendations are given on the evaluation of the uncertainty envelope. The frequency response functions were measured at the tool tip 30 times in each direction, and the variance of the measurements was calculated. The robust stability lobe diagrams were determined for uncertainty levels $1 \sigma, 2 \sigma$ and $3 \sigma$. The results were validated from two aspects. First, numerically generated FRFs were tested in order to validate the conservativeness of the mathematical method. It was found that the predicted and real robust boundaries show good agreement. Second, the real measured FRFs were considered and the stability lobe diagrams were obtained for 100 different combinations. The case study revealed that the uncertainty level $1 \sigma$ gives a reasonable ap- proximation of the true robustness. In case of even more rigorous conditions, the $2 \sigma$ level is recommended, however, it might be unnecessarily conservative.

\section{Acknowledgement}

The research leading to these results has received funding from the European Research Council under the European Unions Seventh Framework Programme (FP/2007-2013) / ERC Advanced Grant Agreement n. 340889 and it was supported by the Hungarian Scientific Research Fund - OTKA PD-112983 and the Janos Bolyai Research Scholarship of the Hungarian Academy of Sciences.

Supported by the ÚNKP-16-3-I. New National Excellence Program of the Ministry of Human Capacities.

\section{Appendix}

\section{Computational algorithm}

If the input data are known, then the robustness of a single parameter point $\left(\Omega_{\mathrm{s}}, a_{\mathrm{p}}\right)$ can be evaluated. The flow chart corresponding to the evaluation process is presented in Fig. 5. In order to calculate the whole stability lobe diagram, the same process has to be repeated. To refine the boundaries, a bisection method can be utilized according to [37].

\section{Comparison with conventional method}

The $\mu$-analysis introduced by [23] bounds the exact $\mu$-value from above and below by the limits

$$
\max _{\mathbf{U} \in \mathcal{U}} \rho(\mathbf{U M}) \leq \mu(\mathbf{M}) \leq \inf _{\mathbf{D} \in \mathcal{D}} \bar{\sigma}\left(\mathbf{D M D}^{-1}\right),
$$

where

$$
\begin{aligned}
& \mathcal{U}=\left\{\mathbf{U} \in \mathbf{\Delta}: \mathbf{U}^{*} \mathbf{U}=\mathbf{I}\right\}, \\
& \mathcal{D}=\left\{0<\mathbf{D}=\mathbf{D}^{*}: \mathbf{D} \boldsymbol{\Delta}=\mathbf{\Delta} \mathbf{D}\right\} .
\end{aligned}
$$

The corresponding computational algorithms are included in the Matlab $\mu$-Analysis and Synthesis Toolbox [40]. The bounds can be computed by the built-in mussv function.

The comparison of the two approaches is not straightforward, since the structure of the uncertainty is not arbitrary. Here, we perform a comparison for the case when the variations in the cross frequency response functions are neglected. In this case, matrix $\Delta$ becomes purely diagonal, and therefore $\|\boldsymbol{\Delta}\|_{\mathrm{m}}=\bar{\sigma}(\boldsymbol{\Delta})$, while the dimension of the problem and matrix $\mathbf{M}$ are the same in (34) and 50). This way, the conservativeness and computational times can be compared. For the comparison, the same experimental data were used as in Sec.6, with the uncertainty in the cross frequency response functions being set to zero. Note, that if it is not the case, then the dimension has to be increased and matrix $\mathbf{M}$ has to be constructed in a different way.

The results are presented for three different points in Fig. 6 , see point A, B and C. The results are listed in Table 2, where index ' $\mathrm{Tb}$ ' refers to the Matlab Toolbox and ' $\mathrm{K}$ ' refers to the solution given by [34, according to [24]. The results show that the computational time is much smaller in the proposed method, while the accuracy is still remarkably good. 


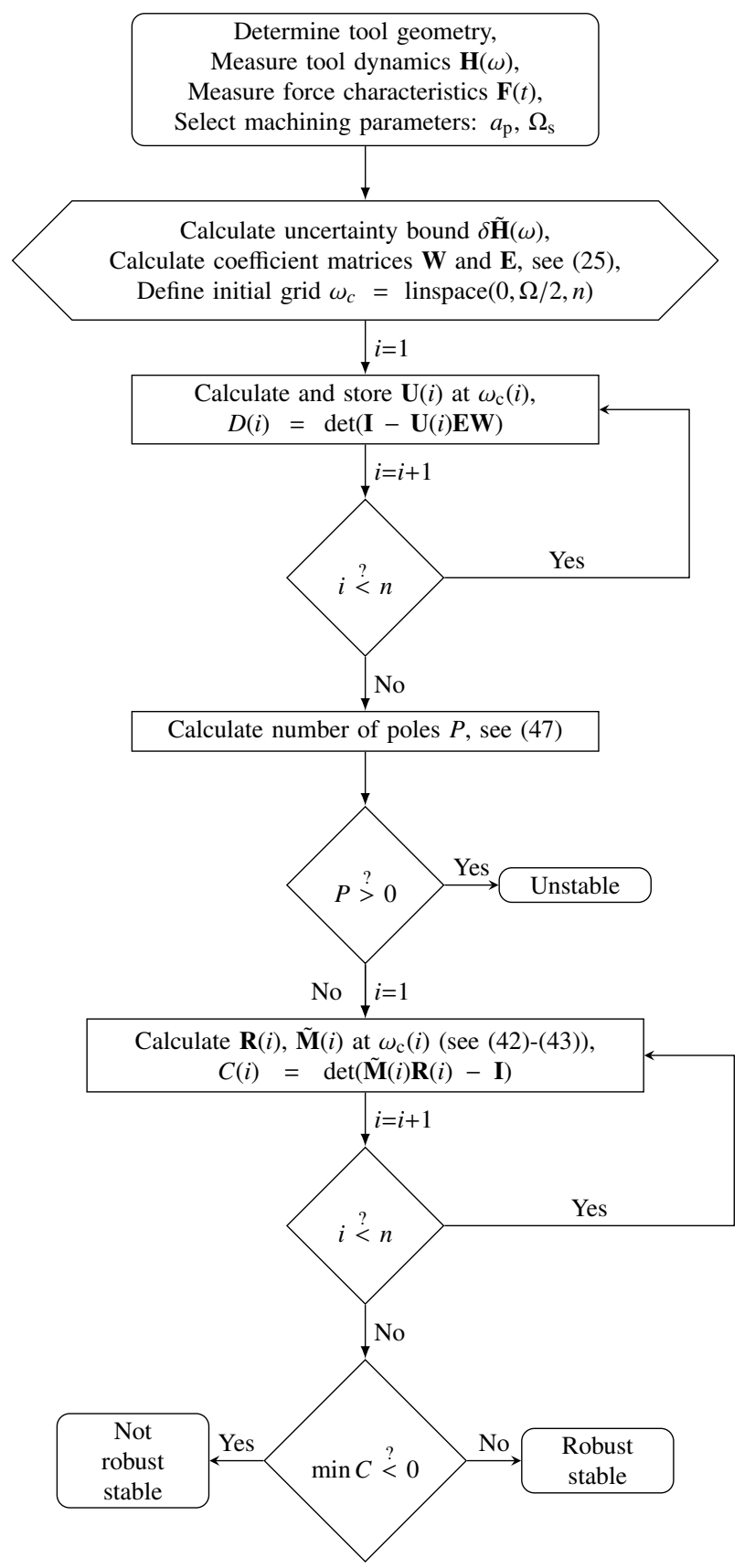

Figure 5: Flowchart for the calculation of robustness.

\section{Bibliography}

[1] S. Tobias, Machine-tool Vibration, Blackie, Glasgow, 1965.

[2] J. Tlusty, L. Spacek, Self-excited vibrations on machine tools, Nakl. CSAV, Prague. in Czech., 1954.

[3] Y. Altintas, E. Budak, Analytical prediction of stability lobes in milling, CIRP AnnManuf Techn 44 (1995) 357-362.

[4] E. Budak, Y. Altintas, Analytical prediction of chatter stability in milling, part i: General formulation, J Dyn SystT ASME 120 (1998) 22-30.

[5] D. Bachrathy, G. Stepan, Improved prediction of stability lobes with extended multi frequency solution, CIRP Ann - Manuf Techn 62 (2013) 411-414.

[6] T. Insperger, G. Stepan, Semi-discretization method for delayed systems, Int J Numer Meth Eng 55 (2002) 503-518.

[7] T. Insperger, G. Stepan, Semi-discretization for time-delay systems, Vol. 178, Springer, New York, 2011.
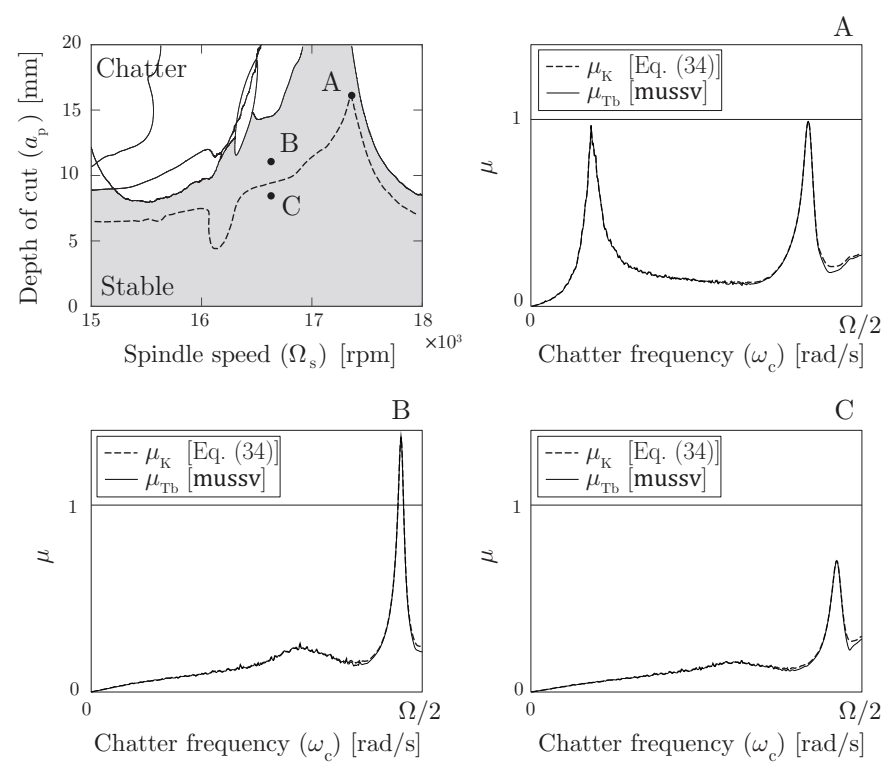

Figure 6: Comparison of the $\mu$-values obtained by 34 and Matlab Toolbox.

Table 2: Comparison of computational times and accuracies.

\begin{tabular}{ccccccc}
\hline & $\Omega_{\mathrm{s}}$ & $a_{\mathrm{p}}$ & $\mu_{\mathrm{Tb}}^{\max }$ & $\mu_{\mathrm{K}}^{\max }$ & time $_{\mathrm{Tb}}$ & time $_{\mathrm{K}}$ \\
& $\mathrm{rpm}$ & $\mathrm{mm}$ & - & - & $\mathrm{s}$ & $\mathrm{s}$ \\
\hline $\mathrm{A}$ & 1736 & 16.2 & 0.990 & 0.991 & 88.28 & 0.49 \\
$\mathrm{~B}$ & 1660 & 11.0 & 1.361 & 1.365 & 14.41 & 0.58 \\
$\mathrm{C}$ & 1660 & 8.0 & 0.700 & 0.703 & 24.59 & 0.47 \\
\hline
\end{tabular}

[8] Y. Ding, L. M. Zhu, X. J. Zhang, H. Ding, A full-discretization method for prediction of milling stability, Int J Mach Tool Manu 50 (2010) 502-509.

[9] X. J. Zhang, C. H. Xiong, Y. Ding, M. J. Feng, Y. L. Xiong, Milling stability analysis with simultaneously considering the structural mode coupling effect and regenarative effect, Int J Mach Tool Manu 53 (2012) 127-140.

[10] M. Zatarain, Z. Dombovari, Stability analysis of milling with irregular pitch tools by the implicit subspace iteration method, Int J Dynam Control 2 (2014) 26-34

[11] E. A. Butcher, O. A. Bobrenkov, E. Bueler, P. Nindujarla, Analysis of milling stability by the chebyshev collocation method: algorithm and optimal stable immersion levels, J Comput Nonlin DynT ASME 4 (2009) 031003.

[12] G. Totis, P. Albertelli, M. Sortino, M. Monno, Efficient evaluation of process stability in milling with spindle speed variation by using the chebyshev collocation method, J Sound Vib 333 (2014) 646-668.

[13] F. A. Khasawneh, B. P. Mann, A spectral element approach for the stability of delay systems, Int J Numer Meth Eng 87 (2011) 566-952.

[14] H. S. Kim, T. Schmitz, Bivariate uncertainty analysis for impact testing, Measurement Science and Technology 18 (2007) 3565-3571.

[15] Z. Mao, M. Todd, Statistical modeling of frequency response function estimation for uncertainty quantification, Mechanical Systems and Signal Processing 38 (2013) 333-345.

[16] S. S. Park, Y. M. Qin, Robust regenerative chatter stability in machine tools, Int J Adv Manuf Technol 33 (2007) 389-402.

[17] E. Graham, M. Mehrpouya, S. S. Park, Robust prediction of chatter stability in milling based on the analytical chatter stability, Journal of Manufacturing Processes 15 (2013) 508-517.

[18] E. Graham, M. Mehrpouya, R. Nagamune, S. S. Park, Robust prediction of chatter stability in micro milling comparing edge theorem and LMI, CIRP Journal of Manufacturing Science and Technology 7 (2014) 29-39.

[19] G. Totis, RCPM - a new method for robust chatter prediction in milling, International Journal of Machine Tools and Manufacture 49 (2009) 273284.

[20] N. D. Sims, G. Manson, B. P. Mann, Fuzzy stability analysis of regen- 
erative chatter in milling, Journal of Sound and Vibration 329 (8) (2009) 1025-1041.

[21] M. Löser, K. Großmann, Influence of parameter uncertainties on the computation of stability lobe diagrams, in: 7th HPC 2016 CIRP Conference on High Performance Cutting, Vol. 46, Procedia CIRP, 2016, pp. 460463.

[22] D. Hajdu, T. Insperger, G. Stepan, Robust stability analysis of machining operations, The International Journal of Advanced Manufacturing Technology 88 (2016) 45-54.

[23] J. Doyle, Analysis of feedback systems with structured uncertainties, IEE Proc. 129, Part D. (1982) 242-250.

[24] M. Karow, D. Hinrichsen, A. J. Pritchard, Interconnected systems with uncertain couplings: explicit formulae for $\mu$-values, spectral value sets and stability radii, SIAM Journal on Control and Optimization 45 (3) (2006) $856-884$

[25] Z. Dombovari, G. Stepan, The effect of helix angle variation on milling stability, Journal of Manufacturing Science and Engineering 134 (5) (2012) 051015.

[26] M. Zatarain, Z. Dombovari, Stability analysis of milling with irregular pitch tools, Int J Dynam Control 2 (2014) 26-34.

[27] V. Sellmeier, B. Denkena, Stable islands in the stability chart of milling processes due to unequal tooth pitch, Int J Mach Tool Manu 51 (2011) $152-164$.

[28] Z. Dombovari, J. Munoa, G. Stepan, General milling stability model for cylindrical tools, Procedia CIRP 4 (2012) 90-97.

[29] Y. Altintas, Manufacturing Automation - Metal Cutting Mechanics, Machine Tool Vibrations and CNC Design, Second Edition, Cambridge University Press, Cambridge, 2012.

[30] D. J. Ewins, Modal Testing, Theory, Practice, and Application, Research Studies Press 2nd edition, 2000.

[31] M. Farkas, Periodic motions, Vol. 104, Springer-Verlag, New York, 1994.

[32] T. Insperger, G. Stepan, Stability chart for the delayed mathieu equation, Proceedings of the Royal Society Mathematical Physical and Engineering Sciences 458 (2024) (2002) 1989-1998.

[33] D. Hinrichsen, A. J. Pritchard, Mathematical Systems Theory I. Modelling, State Space Analysis, Stability and Robustness, Vol. 48, SpringerVerlag, Berlin, 2005.

[34] A. Packard, J. C. Doyle, The complex structured singular value, Automatica 29 (1) (1993) 71-109.

[35] L. Qiu, B. Bernhardsson, A. Rantzer, E. J. Davison, P. M. Young, J. C. Doyle, A formula for computation of the real stability radius, Automatica 31 (6) (1995) 879-890.

[36] M. K. H. Fan, A. L. Tits, J. C. Doyle, Robustness in the presence of mixed parametric uncertainty and unmodeled dynamics, IEEE Transactions on Automatic and Control 36 (1) (1991) 25-38.

[37] D. Bachrathy, G. Stepan, Bisection method in higher dimensions and the efficiency number, Periodica Polytechnica Mechanical Engineering 56 (2) (2012) 81-86.

[38] F. R. Gantmacher, The theory of matrices, Vol. 2, Chelsea Pub. Co., New York, 1959.

[39] G. Stepan, Retarded dynamical systems, Longman, Harlow, 1989.

[40] G. Balas, J. C. Doyle, K. Glover, A. Packard, R. Smith, $\mu$-Analysis and Synthesis Toolbox for Use with Matlab User's Guide, The MathWorks Inc, 1993. 